\title{
A Well Dressed Microscope: Practical Experience with Microcalorimeter and Silicon Drift Detector Systems
}

\author{
John A. Small*, Dale E. Newbury*, John Henry J. Scott*, Lance King*, Sae Woo Nam**, Kent \\ Irwin**, Steve Deiker**, Shaul Barkan,*** and Jan Iwanczyk*** \\ *Surface \& Microanalysis Science Division, NIST, Gaithersburg, MD 20899 \\ $* *$ NIST, EEEL, Boulder, CO 230940 \\ ****Photon Imaging Inc., Northridge, CA 91324
}

NIST, Gaithersburg has recently installed a first generation silicon drift detector (SDD) from Photon Imaging and the NIST Boulder microcalorimeter energy dispersive x-ray spectrometer ( $\mu$ cal-EDS) on a JEOL $840 \mathrm{SEM}^{1}$, as shown in Fig. 1.[1,2] The instrument is also equipped with a conventional $\mathrm{Si}$-Li X-ray detector (LINK ISIS 3 position turret) and a JEOL wavelength dispersive x-ray spectrometer. NIST, Gaithersburg staff have had the opportunity to work with these new detector technologies as "users" and to compare preliminary results with conventional systems.

$\mu$ cal-EDS: The NIST $\mu$ cal-EDS has been outfitted with a second-generation Mo-Cu sensor with a Bi absorber replacing the first-generation $\mathrm{Al}-\mathrm{Ag}$ sensor and $\mathrm{Bi}$ absorber. We obtained $7 \mathrm{eV}$ resolution on $\mathrm{Al}$ as shown in Fig. 2. The maintenance of the cryogenic systems includes the filling of the $\mathrm{LN}_{2}$ and LHe reservoirs and the magnetization cycle of the adiabatic demagnetization refrigerator (ADR). Although fairly complex, these tasks became routine after only a few operations. A minor drawback of the existing cryostat is the need to fill the $\mathrm{LN}_{2}$ reservoir every day (this is in the process of being automated) and the LHe reservoir (which does not currently lend itself to automation) every other day. At the NIST Gaithersburg labs this requires two people (for safety) to be present during the LHe transfer. After the initial cool down and operation of the detector system, a vacuum leak caused the ADR to warm up to room temperature. The standard procedure for obtaining good performance is to initially cool the detector system in a low magnetic field and attachthe system to a probe after the system is cooled to $4 \mathrm{~K}(\mathrm{LHe})$. However, we attempted to cool the system down while it was attached to the microprobe. Our attempt to obtain at least $10 \mathrm{eV}$ resolution was not successful, and the resolution degraded to about $50 \mathrm{eV}$. There are several possible reasons that are being explored such as inadequate magnetic shielding and ground loops.

SDD Detector: Operation of the SDD was straightforward with similar requirements to operating a conventional Si-Li detector. The Peltier cooler requires about 10 minutes to reach the operational temperature and is then stable indefinitely. Spectra collected with the SDD (resolution of $175 \mathrm{eV}$ at $\mathrm{MnK} \alpha$ for a $4 \mu$ s time constant) are in general comparable to a conventional SiLi detector. One interesting exception is the low-energy response of the SDD, which appears to be significantly better than that of a conventional Si-Li detector with an ultrathin window, as shown in Fig. 3 for Mn. The spectrum from the LINK detector was collected immediately after conditioning to eliminate any ice. The limiting count rate for the SDD appears (from oscilloscope traces) to be on the order of $1.5 \mathrm{MHz}$ to $2.5 \mathrm{MHz}$. However, the peak-processing electronics limit the practical output count rate to about

\footnotetext{
${ }^{1}$ Certain commercial equipment, instruments, or materials are identified in this abstract to specify adequately the experimental procedure. Such identification does not imply recommendation or endorsement by the National Institute of Standards and Technology, nor does it imply that the materials or equipment identified are necessarily the best available for the purpose.
} 
$100 \mathrm{kHz}$. To take full advantage of the detector's counting speed will require a redesign of these electronics.

References

[1] D.A. Wollman, et al., Nucl. Instrum. Meth. A 444:(1-2) (2000) 145-150.

[2] B.E. Patt, J.S.Iwanczyk, C.R. Tull et al., Microsc. Mioroanal. 6 (Suppl. 2) (2001) 728.

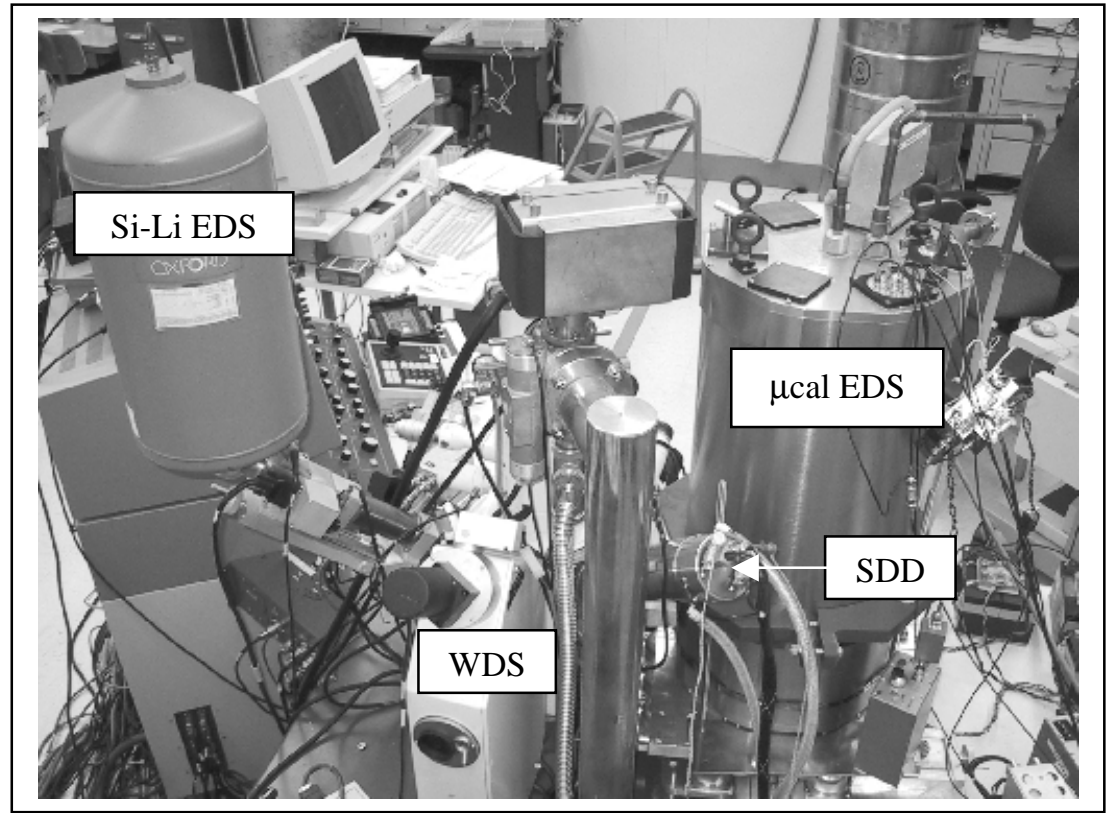

FIG 1. Image of JEOL 840 SEM showing various x-ray detectors.

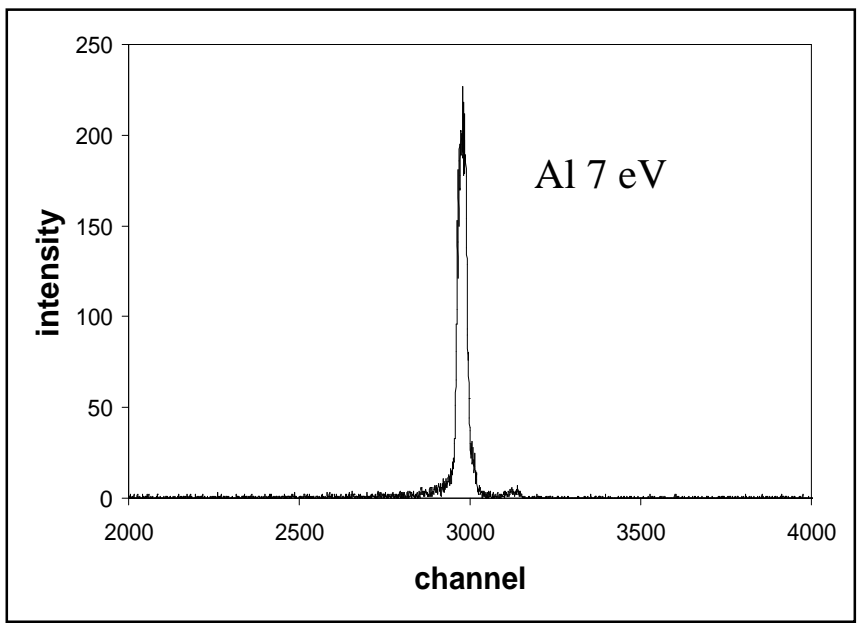

FIG. 2. Al Spectrum from NIST $\mu$ cal EDS.

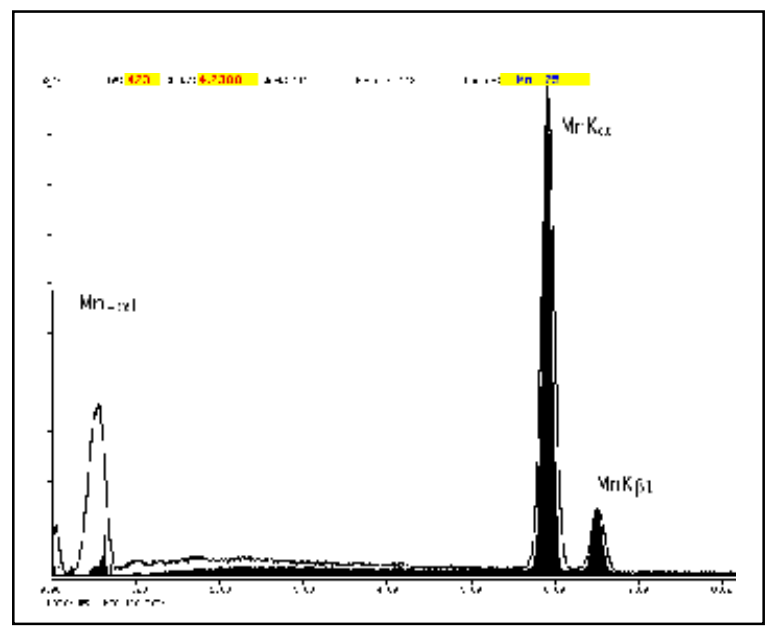

FIG. 3. Mn spectra from the SDD and an ultra thin window Si-Li detector. 